\section{Mortalidade por neoplasia maligna do fígado e vias biliares intra-hepáticas no Brasil, 1980-2010}

\author{
Mortality due to malignant neoplasms of the liver \\ and intrahepatic bile ducts in Brazil, 1980-2010
}

\author{
Mortalidad por neoplasia maligna de hígado y vías \\ biliares intrahepáticas en Brasil, 1980-2010
}

\begin{abstract}
1 Secretaria de Vigilância em Saúde, Ministério da Saúde, Brasília, Brasil.

2 Faculdade de Ciências da Saúde, Universidade de Brasília, Brasília, Brasil.

Correspondência T. R. Amorim Departamento de DST, AIDS e Hepatites Virais, Secretaria de Vigilância em Saúde, Ministério da Saúde. Setor de Administração Federal Sul, Trecho 2, Lote 5/6, Bl. F, Torre I, Edifício Premium, Brasília, $D F$ 70070-600, Brasil.

thiago.sesdf@gmail.com
\end{abstract}

\section{Abstract}

This study aimed to analyze trends in mortality due to malignant neoplasms of the liver and intrahepatic bile ducts in Brazil from 1980 to 2010. This was a time series study using data provided by the Mortality Information System. Crude and standardized mortality rates were calculated according to age, area of residence, and gender. Trends were analyzed using polynomial regression models. An increasing trend in mortality was observed for both males and females in Brazil. Mean overall mortality was 3.59, with a linear increase of $0.020\left(R^{2}=0.588 ; p<0.001\right)$. The rate for males was 4.20 deaths per 100,000 inhabitants with a linear annual increase of $0.044\left(R^{2}=0.81 ; p<0,001\right)$, and for females, 2.98 per 100,000 inhabitants with a linear annual increase of $0.0194\left(R^{2}=0.35 ; p=0.008\right)$. The article discusses possible explanations for this increase, as well as potential information bias.

Hepatocellular Carcinoma; Neoplasms; Mortality
Thiago Rodrigues de Amorim 1

Edgar Merchán-Hamann ${ }^{2}$

\section{Resumo}

O objetivo deste trabalho foi analisar a tendência da mortalidade por neoplasia maligna do figado e das vias biliares intra-hepáticas no Brasil, entre 1980 e 2010. Trata-se de um estudo de séries temporais com dados do Sistema de Informações sobre Mortalidade. Coeficientes de mortalidade brutos, padronizados, específicos por idade, região de residência e sexo foram calculados e as tendências analisadas utilizando-se modelos de regressão polinomial. Tendência crescente da mortalidade foi observada no Brasil, para ambos os sexos. O coeficiente médio de mortalidade para o país foi de 3,59 óbitos por $100 \mathrm{mil}$ habitantes com aumento linear anual de 0,020 $\left(R^{2}=0,588 ; p<0,001\right)$, sendo para o sexo mas culino de 4,20 óbitos por 100 mil homens, com aumento linear de 0,044 $\left(R^{2}=0,81 ; p<0,001\right)$ ao ano e, para o sexo feminino, de 2,98 por 100 mil mulheres, com aumento de 0,0194 $\left(R^{2}=0,35\right.$; $p=0,008$ ao ano. Discutem-se possíveis causas desse aumento bem como vieses de informação.

Carcinoma Hepatocelular; Neoplasias; Mortalidade 


\section{Introdução}

As neoplasias malignas do fígado e das vias biliares intra-hepáticas representam, em conjunto, a terceira causa de óbito por câncer no mundo, ocupando a quinta posição entre os tipos mais comuns nos indivíduos do sexo masculino e a sétima nos do sexo feminino. A maioria dos casos (cerca de 85\%) ocorre nos países em desenvolvimento, particularmente no sexo masculino e em determinadas regiões da África Subsaariana e do sudeste da Ásia. Estimou-se para o ano de 2008 a ocorrência de quase 694 mil óbitos no mundo em decorrência dessas neoplasias, sendo 477 mil em homens e 217 mil em mulheres 1.

No Brasil, entre os anos de 1979 e 2008, o câncer de fígado esteve na sétima posição entre as causas de óbito por câncer entre as mulheres, ao passo em que, nos homens, ele passou da oitava colocação no período de 1979 a 1983 para a sexta entre 2004 e 2008 2. Nos anos subsequentes, 2009 e 2010, o número de óbitos registrados foi de 7.580 e 7.721 , o que representou $4,4 \%$ e $4,3 \%$ dos óbitos entre todas as neoplasias malignas (Departamento de Informática do SUS. http:// tabnet.datasus.gov.br/cgi/tabcgi.exe?sim/cnv/ obt10uf.def, acessado em 16/Nov/2012).

Embora com distribuição variável no mundo, nas populações com elevada ocorrência desse tipo de câncer os principais fatores de risco são a infecção crônica pelo vírus da hepatite B (VHB) e a exposição à aflatoxina B1. De outro modo, em locais onde se evidencia baixa ocorrência, especialmente nos países desenvolvidos, o consumo de álcool e a infecção pelo vírus da hepatite $\mathrm{C}$ (VHC) comparecem como fatores de risco mais importantes 3 .

A prevalência dos fatores de risco no Brasil é variável. Os resultados de um estudo de base populacional realizado no conjunto das capitais brasileiras (população de 20 a 69 anos) classificam o país como de baixa endemicidade para as hepatites virais dos tipos $\mathrm{B}$ e $\mathrm{C}$, com prevalências estimadas em $0,6 \%$ e $1,6 \%$, respectivamente ${ }^{4,5,6}$. Quanto ao uso do álcool, $18 \%$ da população adulta brasileira apresentam consumo abusivo, sendo este mais elevado para o sexo masculino, em jovens e indivíduos com maior escolaridade 7 .

As condições climáticas no Brasil favorecem, em grande medida, o crescimento de fungos produtores de micotoxinas, dentre as quais se destacam as aflatoxinas 8 . Devido aos seus efeitos carcinogênicos, a exposição humana a tais substâncias representa um fator importante a ser considerado na etiologia do câncer hepático. Há evidências de contaminação dos alimentos no país, especialmente o milho em grão, o amen- doim e seus derivados, a castanha-do-pará, o leite, entre outros $8,9,10,11$.

Dentre as estratégias de prevenção e controle disponíveis citam-se a vacinação contra a hepatite $\mathrm{B}$, a triagem sorológica para as hepatites virais nos doadores de sangue, o diagnóstico precoce e o tratamento dos portadores de hepatites virais crônicas, a triagem do câncer de fígado em grupos vulneráveis utilizando-se a alfa-fetoproteína sérica e marcadores virais, a ultrassonografia e outras técnicas radiológicas e, também, as legislações para o controle de micotoxinas em alimentos de origem animal e vegetal.

Face ao exposto, o objetivo deste trabalho foi analisar a tendência temporal da mortalidade por neoplasia maligna do fígado e vias biliares intra-hepáticas no Brasil, compreendendo o período de 1980-2010.

\section{Métodos}

Realizou-se uma análise de séries temporais, cuja unidade de observação foi constituída pelos óbitos por neoplasia maligna do fígado e vias biliares intra-hepáticas ocorridos no Brasil entre os anos de 1980 e 2010. Os dados foram obtidos com base nos arquivos de declarações de óbito não nominais provenientes do Sistema de Informações sobre Mortalidade (SIM) e que estão disponíveis no sítio do DATASUS (http://tabnet.datasus.gov. br/tabdata/sim/dados/cid10_indice.htm, acessado em 12/Nov/2011). Foi realizado o download dos arquivos de cada Unidade Federada, referente aos óbitos não fetais de indivíduos residentes ocorridos em cada ano (1980 a 2010). Ao todo, foram 423 arquivos disponibilizados para os anos de 1980-1995 e 405 arquivos de 1996-2010, totalizando 828. Utilizou-se o programa Tab para Windows (Tabwin versão 3.5. Departamento de Informática do SUS. http://portal.saude.gov.br/ portal/se/datasus/area.cfm?id_area=7325) para expandir os arquivos compactados do formato “.DBC” para “.DBF”. Em seguida, reuniram-se todos os arquivos utilizando o SPSS 18.0 (SPSS Inc., Chicago, Estados Unidos) conformando uma única base de dados. Desse ponto em diante selecionaram-se as variáveis de interesse, quais sejam: data do óbito, data de nascimento, idade, sexo, código do município de residência e causa básica de óbito (com até 4 dígitos). Com base nessas variáveis obteve-se a região de residência, o ano do óbito e as faixas etárias.

Os códigos da Classificação Internacional de Doenças (CID) utilizados referiram-se à causa básica de óbito, na 9a revisão 155 (que inclui as subcategorias 155.0, 155.1 e 155.2) e, na 10a revisão, C22 (que inclui as subcategorias C22.0, 
C22.1, C22.2, C22.3, C22.4, C22.7, C22.9). Para o cálculo dos coeficientes de mortalidade utilizouse como denominador as estimativas populacionais anuais do Instituto Brasileiro de Geografia e Estatística (IBGE), por sexo, idade e região de residência. Foram constituídos seis grupos etários para a análise, a saber: 0-39 anos, 40-49, 50-59, 60-69, 70-79 anos e 80 e mais anos.

Coeficientes brutos de mortalidade por 100 mil habitantes foram calculados e, posteriormente, padronizados pelo método direto utilizando a população mundial proposta por Segi 12 e modificada por Doll et al. 13. Coeficientes específicos de mortalidade por sexo, faixa etária e região de residência foram calculados, bem como a mortalidade proporcional por neoplasia de fígado e vias biliares intra-hepáticas entre todos os óbitos ocorridos no período. Exibe-se a série temporal agrupada em um primeiro período abrangendo seis anos, seguida de mais cinco quinquênios, dispostos do seguinte modo: 1980-1985, 1986-1990, 1991-1995, 1996-2000, 2001-2005 e 2006-2010.

Para explorar a tendência de mortalidade empregou-se a técnica de regressão linear simples, com a qual se avaliou a tendência da razão dos coeficientes de mortalidade entre os sexos masculino e feminino e a mortalidade proporcional. Para as tendências entre as faixas etárias empregaram-se modelos de regressão polinomial 14. Iniciou-se o estudo de regressão a partir da construção de gráficos de dispersão dos pontos, considerando como variável dependente (y) o coeficiente de mortalidade, e como variável independente $(\mathrm{x})$ a variável ano centralizada pelo ponto médio do período (x-1995). Daí em diante, modelos de regressão linear simples, de segundo e terceiro graus foram testados. A eleição do modelo mais adequado seguiu os critérios de melhor ajuste do coeficiente de determinação (R²), da análise dos resíduos e da significância estatística do modelo (considerando o nível de $5 \%$ de significância), e quando estas se assemelharam, decidiu-se pelo modelo mais simples.

Para o cálculo das taxas de mortalidade foi utilizado o programa Excel 2007 (Microsoft Corp., Estados Unidos) e para as análises de tendência o PASW 18.0 (IBM, Nova York, Estados Unidos). O projeto foi aprovado pelo comitê de ética em pesquisa da Faculdade de Ciências da Saúde da Universidade de Brasília, sob o no 23.036 de 10/ Abr/2012.

\section{Resultados}

Ocorreram 139.530 óbitos por neoplasia maligna do fígado e vias biliares intra-hepáticas no Bra- sil, entre os anos de 1980 e 2010. Destaca-se que $65,8 \%$ (91.769) desses foram classificados como neoplasia maligna do fígado não especificada (subcategorias 155.2 e C22.9) e $21 \%$ (29.378) como carcinoma hepatocelular. Segundo o gênero, $54,5 \%$ (76.045) dos óbitos aconteceram no sexo masculino e $84,7 \%$ (118.156) em indivíduos com idades iguais ou superiores a 50 anos. Dentre o sexo masculino a maior proporção de óbitos (27,9\%) ocorreu na faixa etária de 60 a 69 anos, enquanto que no sexo feminino a maior proporção $(26,7 \%)$ foi encontrada na faixa etária entre 70 e 79 anos. A mortalidade proporcional por neoplasia maligna do fígado e vias biliares intrahepáticas exibiu tendência de crescimento $\left(\mathrm{R}^{2}=\right.$ 0,$94 ; \mathrm{p}<0,001$ ), variando de $0,36 \%$ a $0,69 \%$, entre 1980 e 2010.

Na Figura 1, pode-se observar uma tendência crescente da mortalidade por neoplasia maligna do fígado e vias biliares intra-hepáticas no Brasil, mas com diferenças entre os sexos. $\mathrm{O}$ coeficiente médio para o país foi de 3,59 com aumento linear anual de $0,020\left(R^{2}=0,588 ; p<0,001\right)$, enquanto que, para o sexo masculino, os coeficientes padronizados de mortalidade foram mais elevados, em toda série histórica, registrando um coeficiente médio de 4,20 óbitos por 100 mil homens, com aumento linear de 0,044 ao ano. Para o sexo feminino, o coeficiente médio de mortalidade foi de 2,98 por 100 mil mulheres, com aumento linear de 0,0194 ao ano. A razão entre os coeficientes de mortalidade padronizados masculino e feminino foi, em média, 1,4 com aumento linear de 0,014 ao ano $\left(R^{2}=0,88 ; p<0,001\right)$.

Na Tabela 1, observa-se que os coeficientes de mortalidade específicos por idade aumentaram com a progressão das faixas etárias, e são ainda mais pronunciados para indivíduos com idades superiores a 50 anos. Considerando o primeiro e o último períodos, houve discreta queda dos coeficientes de mortalidade para as faixas etárias de 0 a 39 anos $(-4,35 \%)$ e 40 a 49 anos $(-6,64 \%)$ e aumento nas demais, especialmente naquela representada por 80 anos e mais, cujo aumento foi cerca de $27 \%$.

De acordo com a Tabela 2, as regiões Norte, Nordeste e Centro-oeste foram as que exibiram crescimento percentual no coeficiente de mortalidade comparando-se o período de 1980-1985 e 2006-2010, e, dentre estas, a Nordeste registrou a maior variação com aumento de $96,42 \%$, enquanto Norte e Centro-oeste mostraram aumento dos coeficientes igual a $21,97 \%$ e $15,19 \%$, respectivamente. Em contrapartida, uma ligeira redução percentual nos coeficientes de mortalidade se deu nas regiões Sudeste $(-6,83 \%)$ e Sul e $(-1,33 \%)$. Com relação ao sexo masculino, os coeficientes de mortalidade aumentaram em 
Coeficientes padronizados de mortalidade (por 100 mil habitantes) por neoplasia maligna do fígado e vias biliares intrahepáticas, segundo sexo. Brasil, 1980-2010.

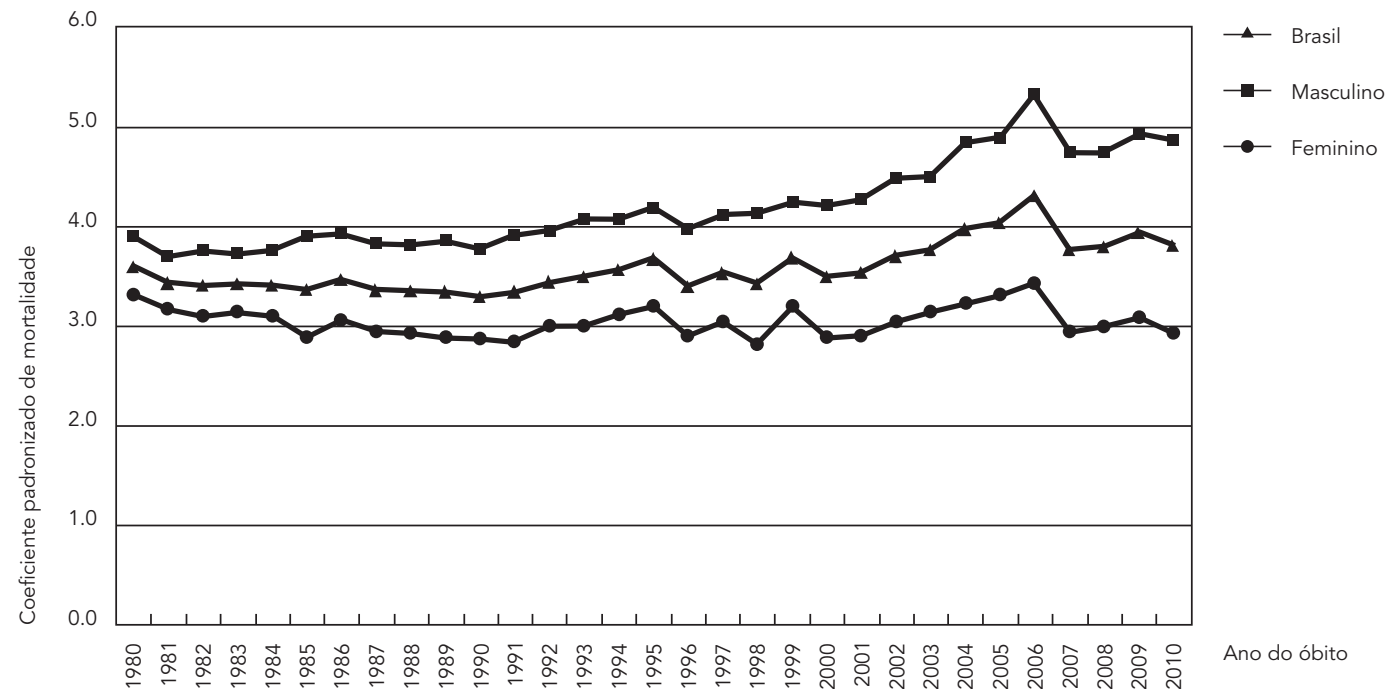

Masculino - modelo: $Y=4,207+0,044(x-1995)$; tendência: crescente $\left(R^{2}=0,81\right.$ e $\left.p<0,001\right)$

Feminino - modelo: $Y=2,987+0,0194(x-1995)+0,0007(x-1995)^{2}-0,0001(x-1995) 3$; tendência: crescente $\left(R^{2}=0,35\right.$ e $\left.p=0,008\right)$.

todas as regiões, com maior intensidade na Nordeste $(126,51 \%)$ e menor na Sudeste $(6,24 \%)$ e, nas demais, o aumento foi em média de $25 \%$ nos coeficientes do primeiro e último períodos. No sexo feminino, os incrementos nos coeficientes ocorreram nas regiões Norte $(18,85 \%)$, Nordeste (71,93\%) e Centro-oeste $(0,91 \%)$, e o decréscimo se deu nas regiões Sudeste $(-19,87 \%)$ e Sul $(-22,7 \%)$.

Quanto à tendência de mortalidade por sexo segundo faixas etárias (Tabela 3), observou-se que para ambos os sexos com idades entre 0 e 39 anos ela foi estável ao longo do período. Notouse tendência decrescente dos coeficientes apenas no sexo feminino nas faixas etárias de 40-49 anos e 50-59 e, a partir destas, houve tendência de crescimento significativo. No sexo masculino, à exceção da faixa etária de 0-39 anos, todas exibiram tendência de crescimento significativo dos coeficientes de mortalidade por neoplasia maligna do fígado e vias biliares intra-hepáticas.

\section{Discussão}

A tendência de mortalidade por neoplasia maligna do fígado e vias biliares intra-hepáticas no Brasil foi crescente ao longo das últimas três décadas, para ambos os sexos. Contudo, tanto o coeficiente médio de mortalidade padronizado quanto o aumento linear anual foram mais pronunciados para o sexo masculino, em todo o tempo. Os mais elevados coeficientes específicos de mortalidade predominaram na faixa etária igual ou superior a 50 anos. Comparando-se os períodos de 1980-1985 e 2006-2010, à exceção das regiões Sudeste e Sul, as demais apresentaram aumento de seus coeficientes de mortalidade, especialmente a Nordeste. Esse mesmo padrão foi observado para o sexo feminino, enquanto para o masculino houve aumento em todas as regiões.

Dentre as medidas de ocorrência nos estudos com neoplasias malignas, a incidência reflete de forma direta as exposições carcinogênicas na população, sendo a mortalidade consequência desta, bem como de seu prognóstico 15. Embora a incidência seja, teoricamente, uma medida de risco mais robusta, o uso de informações sobre a mortalidade pode dar uma dimensão aproxi- 
Tabela 1

Coeficientes de mortalidade (por 100 mil habitantes) brutos, padronizados e específicos de acordo com a faixa etária, por neoplasia maligna do fígado e vias biliares intra-hepáticas. Brasil, 1980-2010.

\begin{tabular}{llccccccc}
\hline Ano do óbito & Bruto & Padronizado & \multicolumn{5}{c}{ Específico por faixa etária (anos) } \\
& & & $\mathbf{0 - 3 9}$ & $\mathbf{4 0 - 4 9}$ & $\mathbf{5 0 - 5 9}$ & $\mathbf{6 0 - 6 9}$ & $\mathbf{7 0 - 7 9}$ & $\mathbf{8 0}$ e mais \\
\hline $1980-1985$ & 2,25 & 3,45 & 0,23 & 2,71 & 7,61 & 15,32 & 28,14 & 36,43 \\
$1986-1990$ & 2,36 & 3,37 & 0,23 & 2,46 & 7,18 & 15,20 & 27,74 & 37,54 \\
$1991-1995$ & 2,54 & 3,51 & 0,21 & 2,46 & 7,24 & 16,12 & 29,81 & 39,91 \\
$1996-2000$ & 2,78 & 3,51 & 0,22 & 2,49 & 7,28 & 16,17 & 28,86 & 41,57 \\
$2001-2005$ & 3,20 & 3,81 & 0,22 & 2,69 & 7,98 & 17,57 & 31,86 & 43,29 \\
$2006-2010$ & 3,79 & 3,93 & 0,22 & 2,53 & 8,51 & 18,09 & 32,71 & 46,25 \\
\hline
\end{tabular}

Tabela 2

Coeficientes padronizados de mortalidade (por 100 mil habitantes) por neoplasia maligna do fígado e vias biliares, segundo região e sexo. Brasil, 1980-2010.

\begin{tabular}{|c|c|c|c|c|c|c|}
\hline \multirow{2}{*}{$\begin{array}{l}\text { Região/Coeficiente de } \\
\text { mortalidade }\end{array}$} & \multicolumn{6}{|c|}{ Período } \\
\hline & $1980-1985$ & $1986-1990$ & 1991-1995 & $1996-2000$ & 2001-2005 & 2006-2010 \\
\hline \multicolumn{7}{|l|}{ Norte } \\
\hline Total & 3,17 & 3,33 & 3,50 & 3,42 & 3,74 & 3,86 \\
\hline Masculino & 3,60 & 3,66 & 3,83 & 3,91 & 4,22 & 4,50 \\
\hline Feminino & 2,71 & 2,95 & 3,15 & 2,92 & 3,23 & 3,22 \\
\hline \multicolumn{7}{|l|}{ Nordeste } \\
\hline Total & 1,98 & 2,05 & 2,29 & 2,48 & 3,20 & 3,88 \\
\hline Masculino & 1,95 & 2,17 & 2,47 & 2,58 & 3,44 & 4,42 \\
\hline Feminino & 2,00 & 1,94 & 2,11 & 2,38 & 2,99 & 3,43 \\
\hline \multicolumn{7}{|l|}{ Sudeste } \\
\hline Total & 4,06 & 3,89 & 3,96 & 3,78 & 3,90 & 3,79 \\
\hline Masculino & 4,66 & 4,59 & 4,72 & 4,69 & 4,96 & 4,95 \\
\hline Feminino & 3,53 & 3,28 & 3,32 & 3,03 & 3,02 & 2,83 \\
\hline \multicolumn{7}{|l|}{ Sul } \\
\hline Total & 4,49 & 4,35 & 4,41 & 4,45 & 4,60 & 4,43 \\
\hline Masculino & 4,92 & 5,01 & 5,14 & 5,35 & 5,76 & 5,94 \\
\hline Feminino & 4,07 & 3,76 & 3,77 & 3,68 & 3,61 & 3,15 \\
\hline \multicolumn{7}{|l|}{ Centro-oeste } \\
\hline Total & 3,39 & 3,19 & 3,55 & 3,96 & 3,95 & 3,90 \\
\hline Masculino & 3,60 & 3,42 & 3,86 & 4,44 & 4,55 & 4,65 \\
\hline Feminino & 3,16 & 2,89 & 3,20 & 3,46 & 3,35 & 3,19 \\
\hline
\end{tabular}

mada da incidência, principalmente neste caso em que a razão mortalidade/incidência para as neoplasias de fígado e vias biliares intra-hepáticas é igual a 0,96 1,16.

Os maiores coeficientes de mortalidade no sexo masculino são corroborados por diversos estudos sobre o padrão de ocorrência do câncer de fígado 1,2,3,17,18,19,20. De modo geral, em todo o mundo, a incidência desse tipo de câncer obtida por meio de registros de câncer é mais elevada para os homens, fato este que ocorre mais acentuadamente em países considerados de baixa incidência, como é o caso do Brasil 2,3. Além disso, nos países em desenvolvimento a prevalência de neoplasias malignas, como um todo, é cerca de $25 \%$ maior em mulheres, revelando o predo- 
Modelos de regressão para os coeficientes de mortalidade por neoplasia maligna do fígado e vias biliares intra-hepáticas, segundo sexo e faixa etária. Brasil, 1980-2010.

\begin{tabular}{|c|c|c|c|c|}
\hline $\begin{array}{l}\text { Sexo/Faixa etária } \\
\text { (anos) }\end{array}$ & Modelo & $\mathbf{R}^{2}$ & Valor de $\mathrm{p}$ & Tendência \\
\hline \multicolumn{5}{|l|}{ Masculino } \\
\hline $0-39$ & $Y=0,252-0,001(x-1995)$ & 0,112 & 0,065 & Não significante \\
\hline $40-49$ & $Y=2,913+0,0305(x-1995)+0,0009(x-1995)^{2}-0,0002(x-1995)^{3}$ & 0,322 & 0,014 & Crescente \\
\hline $50-59$ & $Y=9,248+0,106(x-1995)$ & 0,563 & $<0,001$ & Crescente \\
\hline $60-69$ & $Y=19,802+0,231(x-1995)$ & 0,834 & $<0,001$ & Crescente \\
\hline 70-79 & $Y=34,199+0,391(x-1995)$ & 0,787 & $<0,001$ & Crescente \\
\hline 80 e mais & $Y=43,316+0,644(x-1995)$ & 0,715 & $<0,001$ & Crescente \\
\hline \multicolumn{5}{|l|}{ Feminino } \\
\hline $0-39$ & $Y=0,189-0,0002(x-1995)$ & 0,012 & 0,559 & Não significante \\
\hline $40-49$ & $Y=2,091-0,0074(x-1995)+0,0008(x-1995)^{2}$ & 0,210 & 0,037 & Decrescen-te \\
\hline $50-59$ & $Y=6,127-0,025(x-1995)$ & 0,250 & 0,004 & Decrescen-te \\
\hline $60-69$ & $Y=13,178+0,124(x-1995)+0,002(x-1995)^{2}-0,001(x-1995)^{3}$ & 0,281 & 0,028 & Crescente \\
\hline $70-79$ & $Y=25,479+0,184(x-1995)+0,009(x-1993,5)^{2}-0,001(x-1995)^{3}$ & 0,248 & 0,049 & Crescente \\
\hline 80 e mais & $Y=38,889+0,196(x-1995)$ & 0,304 & 0,001 & Crescente \\
\hline
\end{tabular}

mínio de tipos de câncer com pior sobrevida no sexo masculino, a exemplo do de fígado, esôfago e estômago em homens 17.

Os mais elevados coeficientes de mortalidade observados nos idosos podem ser explicados pelas profundas mudanças no padrão de mortalidade entre os grupos de idade. No período compreendido entre os anos de 1980 e 2001, registrou-se um aumento de $87 \%$ nos óbitos em indivíduos acima de 60 anos ${ }^{21}$ e, nestes, os óbitos relacionados às neoplasias vêm incidindo mais fortemente devido à maior participação deste grupo etário na estrutura populacional, aliada ao aumento da expectativa de vida ${ }^{22}$. Isso também poderia influenciar a maior variação positiva nos coeficientes de mortalidade observada nas faixas etárias mais velhas. Não obstante, vale ressaltar que a maior proporção de causas de óbitos mal definidas no período de 1980 a 2001 encontravase justamente na faixa etária acima dos 60 anos 21 . A redução dessa proporção poderia contribuir para o aumento dos coeficientes de mortalidade por câncer de fígado observado nesse grupo populacional.

Ainda sobre o fenômeno das causas de óbito mal definidas no país, uma importante redução vem se materializando desde a década de 80 , particularmente nas regiões Norte e Nordeste. Nessas regiões, no ano de 1980, a mortalidade proporcional por causas mal definidas representava $75 \%$, e no ano 2000 este porcentual reduziu para $50 \%$ 21. Em progressiva redução, em
2005, essa proporção na Região Nordeste chegou a $17,2 \%$, valor este similar ao da Região Norte e próximo da média nacional de $10,4 \% 22$. Essa redução traduz uma sensível melhoria da qualidade das informações sobre mortalidade no Brasil, do diagnóstico e codificação das causas de óbito, especialmente nessas regiões. Obedecendo a um padrão diferente, nas regiões Sudeste e Sul evidencia-se uma melhor captação e qualidade das informações sobre mortalidade, enquanto na Região Centro-oeste verifica-se um patamar intermediário 21 . As razões citadas poderiam justificar os maiores incrementos porcentuais observados neste estudo para o coeficiente de mortalidade da Região Nordeste, tomando-se os períodos de 1980-1985 e 2006-2010, quando comparado às demais regiões do país.

Diversos trabalhos sobre a tendência de neoplasias no Brasil 18,23,24 chamam a atenção para padrões de ocorrência marcadamente regionais, com elevados coeficientes de mortalidade em localidades com maior desenvolvimento socioeconômico e redes de atenção à saúde melhor estruturadas, a exemplo das regiões Sudeste e Sul. Com isso, os elevados coeficientes podem refletir a real ocorrência ou resultar do melhor registro ou notificação dos dados. Isso fortaleceria a hipótese de um padrão real de redução do coeficiente de mortalidade por câncer de fígado nas regiões Sudeste e Sul, ressaltando-se a diferença entre os sexos, com declínio dos coeficientes para o feminino, evento este restrito às regiões mencionadas 
e, aumento para o masculino, notório em todas as regiões.

Diferentemente de outros estudos que atribuem o aumento dos casos de câncer de fígado ao aumento da frequência de óbitos por carcinoma hepatocelular ou colangiocarcinoma 4,25 , é difícil avaliar se o incremento dos coeficientes de mortalidade no Brasil também se deve a este fato, pois a maioria dos óbitos na série histórica analisada tem sido codificada como neoplasia maligna do fígado não especificada. A despeito do aumento global do diagnóstico e identificação desses tumores no Brasil, parece persistirem dificuldades inerentes à especificação dos mesmos à luz da CID-10. Resta apontar que a proporção de erros na classificação dos diversos tipos de neoplasias varia conforme o tipo, sendo maior em neoplasias de difícil diagnóstico, dentre as quais se incluem o câncer primário de fígado 26 .

Por assim ser, a exposição de argumentos que expliquem tais variações na mortalidade com base em mudanças na prevalência dos fatores de risco para o câncer de fígado no Brasil deve ser cautelosa. Dentre aqueles reconhecidamente importantes, destaca-se a ocorrência das infecções pelos VHB e VHC, o consumo de álcool e a cirrose hepática. Tais fatores foram evidenciados por pesquisas que estudaram o câncer primário de fígado (carcinoma hepatocelular) realizadas em amostras de conveniência de Centros de Referência no Brasil, em dois momentos distintos, 1997 e 2009 27,28.

Em relação à hepatite $\mathrm{B}$, a Região Norte do país possui classificação de alta endemicidade com prevalência do antígeno de superfície do VHB (HBsAg) superior a $8 \%$ e as demais como intermediária. Para a hepatite $\mathrm{C}$, a classificação sugere endemicidade intermediária com prevalência de anti-VHC na população entre $2,5 \%$ e $10 \% 29$. Entretanto, dados mais recentes obtidos por meio de inquérito populacional realizado no conjunto das capitais do Brasil revelou que o padrão de endemicidade para as hepatites virais B e C é baixo, com soroprevalência do HBsAg de 0,37\% (IC95\%: 0,25\%-0,50\%) e de anti-VHC de 1,4\% (IC95\%: 1,1-1,6) 4,5,6.

Considerando as estimativas de prevalência para as hepatites B e C no país, podem-se constatar mudanças no padrão epidemiológico no conjunto das capitais, evidenciando uma redução destas infecções. A despeito disso, um número importante de indivíduos encontra-se cronicamente infectados e sob risco de desenvolver cirrose e/ou câncer hepático, em especial indivíduos mais velhos, do sexo masculino, de condições socioeconômicas desfavoráveis 4,5,6. Somando-se a esse fato, os dados do Sistema de Informação de Agravos de Notificação mostram um aumento progressivo da detecção de casos pelo sistema de vigilância epidemiológica, em sua maioria indivíduos do sexo masculino e na faixa etária superior a 30 anos, que pode estar relacionado à ampliação da oferta de testes diagnósticos nos serviços de saúde ou até mesmo ser resultado da melhoria da captação de casos 30 .

Dado que as hepatites virais crônicas constituem fatores de risco para o câncer de fígado, as medidas de prevenção e controle a elas direcionadas têm efeitos sobre a mortalidade por este tipo de neoplasia. Dentre as medidas de controle empreendidas no Brasil, a triagem nos bancos de sangue para os marcadores sorológicos da hepatite B foi instituída em 198831 e, posteriormente, em 1993, para a hepatite C 32 , o que ampliou a segurança da transfusão de sangue e hemoderivados em relação à possível transmissão por esta via.

No ano de 1992, a OMS lançou recomendações para que os países incluíssem a vacinação contra a hepatite B em seus programas de imunização 33. O Brasil a instituiu em 1991 na Amazônia Legal, para menores de um ano de idade 34 e, desde então, vem ampliando gradualmente o acesso à vacina para faixas etárias e grupos específicos de forma que, atualmente, é disponibilizada para todos os indivíduos menores de 29 anos de idade.

Além das hepatites virais, a cirrose constitui um fator de risco para neoplasia maligna do fígado e das vias biliares intra-hepáticas. Em um estudo sobre a mortalidade por cirrose em adultos no Brasil, observou-se que a maioria dos óbitos ocorreu nos homens $(80,4 \%)$, cujo risco de morte foi 4,5 vezes maior em relação às mulheres. Dentre as regiões, riscos mais elevados localizaramse no Sudeste e Norte. Nessa última, sugeriu-se como etiologia as hepatites virais B e C 35 . Corroborando esses resultados, um trabalho sobre a mortalidade por hepatite B entre 2000 e 2008 revelou coeficientes mais elevados na Região Norte do país, bem como mortalidade proporcional maior para o sexo masculino 36 .

Em relação ao uso de álcool, estima-se que a dependência atinja $11,2 \%$ dos brasileiros que vivem nas 107 maiores cidades do país, segundo levantamento domiciliar sobre o uso de drogas realizado pelo Centro Brasileiro de Informações sobre Drogas Psicotrópicas (CEBRID). Nesta pesquisa ressaltaram-se diferenças entre os sexos, com maior prevalência para o masculino $(17,1 \%)$ quando comparado ao feminino (5,7\%). Não obstante, a prevalência de dependentes foi maior nas regiões Norte e Nordeste com porcentagens acima dos $16 \% 37,38$.

Desde 2006 é realizada no Brasil a Vigilância de Fatores de Risco e Proteção para Doenças 
Crônicas por Inquérito Telefônico (VIGITEL) implantada em todas as capitais dos 26 estados brasileiros e no Distrito Federal. Dados desse sistema indicam que no conjunto da população adulta das 27 cidades estudadas, o consumo abusivo de bebidas alcoólicas foi de $18 \%$, valor este cerca de três vezes maior em homens $(26,8 \%)$ do que em mulheres (10,6\%). Entre os anos de 2006 e 2010 o consumo abusivo de álcool aumentou entre as mulheres, passando de $8,2 \%$ para $10,6 \%$. Nos homens essa proporção variou de $26 \%$ a $29 \% 7$.

Sobre a contribuição das aflatoxinas para o desenvolvimento de câncer de fígado são bem documentados seus efeitos na sua etiologia 8,11. É crescente o número de publicações dedicadas ao estudo da ocorrência dessas substâncias no país, focalizando principalmente a contaminação alimentar e de rações animais $10 \mathrm{em}$ detrimento das exposições humanas e suas consequências. Existem no país alimentos de origem animal e vegetal com importantes concentrações de aflatoxinas, dos quais se destacam o milho em grão, o amendoim, a castanha-do-pará (extraída na Região Norte e que abastece o mercado interno), o leite e muitos outros 8,9,10,11. O Programa Nacional de Controle de Resíduos e Contaminantes (PNCRC) do Ministério da Agricultura, Pecuária e Abastecimento que tem o objetivo de inspecionar e fiscalizar alimentos de origem vegetal e animal bem como a Agência Nacional de Vigilância Sanitária (ANVISA) estabelecem limites máximos que variam entre 0,5 e $20 \mu \mathrm{g} / \mathrm{kg}$ para aflatoxinas em alimentos e produtos a serem ofertados ao consumidor 39,40 .
Face ao exposto, há argumentos que sustentam a hipótese de que existem condições favoráveis ao aumento da mortalidade, além da melhoria da qualidade da informação, por neoplasia maligna do fígado e vias biliares intrahepáticas, principalmente para os indivíduos do sexo masculino. $\mathrm{O}$ elevado número de pessoas infectadas pelo VHB e VHC, o maior consumo de álcool, bem como o maior acometimento pela cirrose hepática contribuem em grande medida para o referido aumento. Dada a complexidade dos fatores associados à ocorrência desse tipo de neoplasia maligna, estudos de caráter etiológico devem ser implementados no país para avaliar a contribuição desses fatores à mortalidade, particularmente no tocante às aflatoxinas. No mais, pesquisas sobre o impacto das medidas de prevenção e controle são fundamentais para ampliar a efetividade dos programas para detecção precoce. Estratégias de aumento e manutenção de elevadas coberturas vacinais contra a hepatite $\mathrm{B}$ para os grupos indicados, melhoria do acesso à triagem sorológica para as hepatites B e C, diagnóstico precoce e implementação do tratamento evitando a progressão para cirrose e o carcinoma hepatocelular, triagem de indivíduos sob maior risco e estímulo à redução do consumo de álcool devem ser tomados como bases para a efetividade de um programa de prevenção e controle. Além disso, a melhoria da codificação das causas de óbito, particularmente, a especificação do tipo de câncer de fígado dentre as subcategorias da CID-10 favoreceria a melhor compreensão da epidemiologia dessa doença e os determinantes a ela relacionados. 


\section{Resumen}

El objetivo del presente trabajo fue analizar la tendencia de la mortalidad por neoplasia maligna de hígado y vías biliares intrahepáticas en Brasil, entre 1980 y 2010. Se trata de un estudio de series temporales con datos del Sistema de Información sobre Mortalidad. Se calcularon las tasas brutas y estandarizadas de mortalidad específicas por edad, región de residencia y sexo. Se analizaron las tendencias, utilizando modelos de regresión polinomial. Fue observada una tendencia creciente de la mortalidad en el país para ambos sexos. La tasa general de mortalidad fue 3,59 muertes $1100 \mathrm{mil}$ habitantes, mostrando aumento lineal anual de 0,020 $\left(R^{2}=0,588 ; p<0,001\right)$; para el sexo masculino: 4,2 muertes 1100 mil hombres, con aumento lineal anual de 0,044 ( $\left.R^{2}=0,81 ; p<0,001\right)$; para el sexo femenino: 2,98 por 100 mil mujeres, con un aumento anual de 0,0194 $\left(R^{2}=0,35 ; p=0,008\right)$. Se discuten posibles causas de tal aumento, así como sesgos en la información.

Carcinoma Hepatocelular; Neoplasias; Mortalidad

\section{Referências}

1. World Health Organization/International Agency for Research on Cancer. Globocan 2008: cancer incidence, mortality and prevalence worldwide in 2008. http://globocan.iarc.fr/ (acessado em 02/ $\mathrm{Mai} / 2012)$.

2. Instituto Nacional de Câncer José Alencar Gomes da Silva. Atlas de mortalidade por câncer. http:// mortalidade.inca.gov.br/Mortalidade/preparar Modelo00.action (acessado em 30/Abr/2012).

3. Mcglynn KA, Tsao L, Hsing AW, Devesa SS, Fraumeni Jr. JF. International trends and patterns of primary liver cancer. Int J Cancer 2001; 94: 290-6.

4. Núcleo de Pós-graduação, Universidade de Pernambuco. Estudo de prevalência de base populacional das infecções pelos vírus das hepatites A, B e C nas capitais do Brasil. Relatório de Pesquisa. http://www.aids.gov.br/publicacao/2010/estudo de_prevalencia_de_base_populacional_das_iin feccoe_pelos_virus_das_hepatites_b (acessado em 02/Mai/2012)

5. Ximenes RAA, Pereira LMMB, Martelli CMT, Merchán-Hamann E, Stein AT, Figueiredo GM, et al. Methodology of a nationwide cross-sectional survey of prevalence and epidemiological patterns of hepatitis A, B and C infection in Brazil. Cad Saúde Pública 2010; 26:1693-704.

\section{Colaboradores}

T. R. Amorim e E. Merchán-Hamann contribuíram igualmente na concepção, redação, revisão crítica das análises e aprovação da versão para publicação. T. R. Amorim foi responsável pela consolidação da base de dados e análise.
6. Pereira LMMB, Martelli CMT, Merchán-Hamann E, Montarroyos UR, Braga MC, Lima MLC, et al. Population-based multicentric survey of hepatitis $\mathrm{b}$ infection and risk factor differences among three regions in Brazil. Am J Trop Med Hyg 2009; 81: 240-7.

7. Secretaria de Gestão Estratégica e Participativa/ Secretaria de Vigilância em Saúde, Ministério da Saúde. VIGITEL Brasil 2010: vigilância de fatores de risco e proteção para doenças crônicas por inquérito telefônico. Brasília: Ministério da Saúde; 2011.

8. Maziero MT, Bersot LS. Micotoxinas em alimentos produzidos no Brasil. Revista Brasileira de Produtos Agroindustriais 2010; 12:89-99.

9. Caldas ED, Silva SC, Oliveira JN. Aflatoxinas e ocratoxina A em alimentos e riscos para a saúde humana. Rev Saúde Pública 2002; 36: 319-23.

10. Rodríguez-Amaya DB, Sabino M. Mycotoxin research in Brazil: the last decade in review. Brazilian Journal of Microbiology 2002; 33:1-11.

11. Oliveira CAF, Germano PML. Aflatoxinas: conceitos sobre mecanismos de toxicidade e seu envolvimento na etiologia do câncer hepático celular. Rev Saúde Pública 1997; 31:417-24. 
12. Segi M. Cancer mortality for selected sites in 24 countries (1950-57). Sendai: Department of Public Health, Tohoku University of Medicine; 1960.

13. Doll R, Payne P, Waterhouse JAH. Cancer incidence in five continents. v. I. Geneva: Union Internationale Contre le Cancer; 1966.

14. Latorre MRDO, Cardoso MRA. Análise de séries temporais em epidemiologia: uma introdução sobre os aspectos metodológicos. Rev Bras Epidemiol 2001; 4:145-52.

15. Departamento de Análise de Situação de Saúde, Secretaria de Vigilância em Saúde, Ministério da Saúde. Saúde Brasil 2008: 20 anos do Sistema Único de Saúde (SUS) no Brasil. Brasília: Ministério da Saúde; 2009.

16. Instituto Nacional de Câncer. Manual de rotinas e procedimentos para registros de câncer de base populacional. Rio de Janeiro: Instituto Nacional de Câncer; 2006.

17. Pisani P, Bray F, Parkin DM. Estimates of the worldwide prevalence of cancer for 25 sites in the adult population. Int J Cancer 2002; 97:72-81.

18. Pinto FG, Curi PR. Mortalidade por neoplasias no Brasil (1980/1983/1985): agrupamento dos Estados, comportamento e tendências. Rev Saúde Pública $1991 ; 25: 276-81$.

19. Tsukuma H, Tanaka H, Ajiki W, Oshima A. Liver cancer and its prevention. Asian Pac J Cancer Prev 2005; 6:244-50.

20. Howlader N, Noone AM, Krapcho M, Neyman N, Aminou R, Altekruse SF, et al. editors. SEER Cance Statistics Review 1975-2009 (Vintage 2009 Populations). http://seer.cancer.gov/csr/1975_2009_ pops09/ (acessado em 02/Mai/2012).

21. Departamento de Análise de Situação de Saúde, Secretaria de Vigilância em Saúde, Ministério da Saúde. Saúde Brasil 2004: uma análise da situação de saúde. Brasília: Ministério da Saúde; 2004.

22. Instituto Brasileiro de Geografia e Estatística. Indicadores sociodemográficos e de saúde no Brasil Rio de Janeiro: Instituto Brasileiro de Geografia e Estatística; 2009

23. Cervi A, Hermsdorff HHM, Ribeiro RCL. Tendência de mortalidade por doenças neoplásicas em $10 \mathrm{ca}$ pitais brasileiras, de 1980 a 2000. Rev Bras Epidemiol 2005; 8:407-18.

24. Wünsch Filho V, Moncau JE. Mortalidade por câncer no Brasil 1980-1995: padrões regionais e tendências temporais. Rev Assoc Med Bras (1992) 2002; 48 :250-7.

25. Taylor-Robinson SD, Toledano MB, Arora S, Keegan TJ, Hargreaves S, Beck A, et al. Increase in mortality rates from intrahepatic cholangiocarcinoma in England and Wales 1968-1998. Gut 2001; 48: 816-20.

26. Silva IS. Cancer epidemiology: principles and methods. Lyon: International Agency for Research on Cancer; 1999.
27. Gonçalves CS, Pereira FEL, Gayotto LCC. Hepatocellular carcinoma in Brazil: report of a national survey (Florianópolis, SC, 1995). Rev Inst Med Trop São Paulo 1997; 39:165-70.

28. Carrilho FJ, Kikuchi L, Branco F, Gonçalves CS, Mattos AAM, et al. Clinical and epidemiological aspects of hepatocellular carcinoma in Brazil. Clinics 2010; 65:1285-90.

29. World Health Organization. Wkly Epidemiol Rec 2002; 77:41-8.

30. Departamento de DST, Aids e Hepatites Virais, Secretaria de Vigilância em Saúde, Ministério da Saúde. Boletim Epidemiológico Hepatites Virais 2011; Ano II, № 1.

31. Brasil. Lei Federal no 7.649, de 25 de janeiro de 1988. Estabelece a obrigatoriedade do cadastra mento dos doadores de sangue, bem como a realização de exames laboratoriais no sangue coletado, visando a prevenir a propagação de doenças, e dá outras providências. Diário Oficial da União 1988; 27 jan.

32. Ministério da Saúde. Portaria no 1.376, de 19 de novembro de 1993. Aprova alterações na Portaria no 721/GM de 09/08/89, que aprova Normas Técnicas para coleta, processamento e transfusões de sangue, componentes e derivados, e dá outras providências. Diário Oficial da União 1993; 2 dez.

33. World Health Assembly. Resolution WHA 45.17. Immunization and vaccine quality. Geneva: World Health Assembly; 1992.

34. Secretaria de Vigilância em Saúde, Ministério da Saúde. Programa Nacional de Imunizações 30 anos. Brasília: Ministério da Saúde; 2003.

35. Lessa I. Cirrose hepática no Brasil: mortalidade e anos produtivos de vida perdidos precocemente. Bol Oficina Sanit Panan 1996; 121:111-22.

36. Tauil MC, Amorim TR, Pereira GFM, Araújo WN. Mortalidade por hepatite viral B no Brasil, 20002009. Cad Saúde Pública 2012; 28:472-8.

37. Carlini EA, Galduroz JCF, Noto AR, Nappo AS. 1o levantamento domiciliar sobre o uso de drogas psicotrópicas no Brasil: estudo envolvendo as 107 maiores cidades do país-2001. São Paulo: Centro Brasileiro de Informações sobre Drogas Psicotrópicas/Departamento de Psicobiologia, Escola Paulista de Medicina; 2002.

38. Galduróz JCF, Caetano R. Epidemiologia do uso de álcool no Brasil. Rev Bras Psiquiatr 2004; 26 Suppl 1:3-6.

39. Brasil. RDC no 7, de 18 de fevereiro de 2011. Dispõe sobre limites máximos tolerados (LMT) para micotoxinas em alimentos. Diário Oficial da União 2011; 22 fev.

40. Brasil. Instrução Normativa no 42, de 31 de dezembro de 2008. Diário Oficial da União 2009; 5 jan.

Recebido em 09/Jul/2012

Versão final reapresentada em 01/Fev/2013 Aprovado em 01/Mar/2013 